\title{
Maximal Digital Straight Segments and Convergence of Discrete Geometric Estimators
}

\author{
François de Vieilleville ${ }^{1}$, Jacques-Olivier Lachaud ${ }^{1}$, and Fabien Feschet ${ }^{2}$ \\ 1 LaBRI, Univ. Bordeaux 1 \\ 351 cours de la Libération, 33405 Talence, France. \\ \{devieill, lachaud\}@labri.fr \\ 2 LLAIC1, IUT Clermont-Ferrand 1 \\ Campus des Cézeaux, 63172 Aubière Cedex, France \\ feschet@llaic3.u-clermont1.fr
}

\begin{abstract}
Discrete geometric estimators approach geometric quantities on digitized shapes without any knowledge of the continuous shape. A classical yet difficult problem is to show that an estimator asymptotically converges toward the true geometric quantity as the resolution increases. We study here the convergence of local estimators based on Digital Straight Segment (DSS) recognition. It is closely linked to the asymptotic growth of maximal DSS, for which we show bounds both about their number and sizes. These results not only give better insights about digitized curves but indicate that curvature estimators based on local DSS recognition are not likely to converge. We indeed invalidate an hypothesis which was essential in the only known convergence theorem of a discrete curvature estimator. The proof involves results from arithmetic properties of digital lines, digital convexity, combinatorics, continued fractions and random polytopes.
\end{abstract}

\section{Introduction}

Estimating geometric features of shapes or curves solely on their digitization is a classical problem in image analysis and pattern recognition. Some of the geometric features are global: area, perimeter, moments. Others are local: tangents, normals, curvature. Algorithms that performs this task on digitized objects are called discrete geometric estimators. An interesting property these estimators should have is to converge towards the continuous geometric measure as the digitization resolution increases. However, few estimators have been proved to be convergent. In all works, shapes are generally supposed to have a smooth boundary and either to be convex or to have a finite number of inflexion points. The shape perimeter estimation has for instance been tackled in [12]. It proved the convergence of a perimeter estimator based on curve segmentation by maximal DSS. The speed of convergence of several length estimators has also been studied in 4]. Klette and Žunić [11] survey results about the convergence (and the speed of convergence) of several global geometric estimators. They show that discrete moments converge toward continuous moments. 
As far as we know, there is only one work that deals with the convergence of local geometric estimators 3. The symmetric tangent estimator appears to be convergent subject to an hypothesis on the growth of DSS as the resolution increases (see Conjecture 1). The same conjecture entails that a curvature estimator is convergent: it is based on DSS recognition and circumscribed circle computation (see Definition 5).

In this paper, we relate the number and the lengths of DSS to the number and lengths of edges of convex hulls of digitized shapes. Using arguments related to digital convex polygons and a theorem induced by random polytopes theory [1, we estimate the asymptotic behaviour of both quantities. We theoretically show that maximal DSS do not follow the conjecture used in [3]. Experiments confirm our result. The convergence theorem is thus not applicable to digital curves. As a consequence, the existence of convergent digital curvature estimators remains an open problem. The paper is organized as follows. First, we recall some standard notions of digital geometry and combinatoric representation of digital lines, i.e. patterns. The relations between maximal segments and edges of convex digital polygons are then studied to get bounds on maximal segments lengths and number. Finally, the asymptotic behaviour of maximal segments is deduced from the asymptotic behaviour of convex digital polygons. The growth of some DSS is thus proved to be too slow to ensure the convergence of curvature estimation. This theoretical result is further confirmed by experiments. Some proofs are omitted for limited space reason but may be found in [6].

\section{Maximal Digital Straight Segments}

We restrict our study to the geometry of 4-connected digital curves. A digital object is a set of pixels and its boundary in $\mathbb{R}^{2}$ is a collection of vertices and edges. The boundary forms a 4-connected curve in the sense used in the present paper. Our work may easily be adapted to 8-connected curves. In the paper, all the reasoning are made in the first octant, but extends naturally to the whole digital plane. A set of successive points of the digital curve from index $A$ to $B$ by $\left[C_{A} C_{B}\right]$ when no ambiguities are raised.

\subsection{Standard Line, Digital Straight Segment, Maximal Segments}

Definition 1. (Réveillès [15]) The set of points $(x, y)$ of the digital plane verifying $\mu \leq a x-b y<\mu+|a|+|b|$, with $a, b$ and $\mu$ integer numbers, is called the standard line with slope $a / b$ and shift $\mu$.

The standard lines are the 4-connected discrete lines. The quantity $a x-b y$ is called the remainder of the line. The points whose remainder is $\mu$ (resp. $\mu+$ $|a|+|b|-1$ ) are called upper (resp. lower) leaning points. The principal upper and lower leaning points are defined as those with extremal $x$ values. Finite connected portions of digital lines define digital straight segment.

Maximal segments form the longest possible DSS in the curve. They are essential when analyzing digital curves: they provide tangent estimations [7, 14, 
they are used for polygonizing the curve into the minimum number of segments 8. Any point belongs to at least one maximal segment.

\subsection{Patterns and DSS}

We here recall a few properties about patterns composing DSS and their close relations with continued fractions. They constitute a powerful tool to describe discrete lines with rational slopes [2, 9]. Since we are in the first octant, the slopes are between 0 and 1 .

Definition 2. Given a standard line $(a, b, \mu)$, we call pattern of characteristics $(a, b)$ the succession of Freeman moves between any two consecutive upper leaning points. The Freeman moves between any two consecutive lower leaning points define the reversed pattern, it is the previous word read from back to front.

A pattern $(a, b)$ embedded anywhere in the digital plane is obviously a DSS $(a, b, \mu)$ for some $\mu$. Since a DSS contains at least either two upper or two lower leaning points, a DSS $(a, b, \mu)$ contains at least one pattern or one reversed pattern of characteristics $(a, b)$.

Definition 3. We call simple continued fraction and we write:

$$
z=a / b=\left[0, u_{1} \ldots, u_{i}, \ldots, u_{n}\right]
$$

We call $k$-th convergent the simple continued fraction formed of the $k+1$ first partial quotients: $z_{k}=\frac{p_{k}}{q_{k}}=\left[0, u_{1}, \ldots, u_{k}\right]$.

There exists a recursive transformation for computing the pattern of a standard line from the simple continued fraction of its slope [2]. We call $E$ the mapping from the set of positive rational number smaller than one onto Freeman-code's words defined as follows. First terms are stated as $E\left(z_{0}\right)=0$ and $E\left(z_{1}\right)=0^{u_{1}} 1$ and others are expressed recursively:

$$
\begin{aligned}
E\left(z_{2 i+1}\right) & =E\left(z_{2 i}\right)^{u_{2 i+1}} E\left(z_{2 i-1}\right) \\
E\left(z_{2 i}\right) & =E\left(z_{2 i-2}\right) E\left(z_{2 i-1}\right)^{u_{2 i}}
\end{aligned}
$$

In the following, the complexity of a pattern is the depth of its decomposition in simple continued fraction. We recall a few more relations:

$$
\begin{array}{r}
p_{k} q_{k-1}-p_{k-1} q_{k}=(-1)^{k+1} \\
\left(p_{k}, q_{k}\right)=u_{k}\left(p_{k-1}, q_{k-1}\right)+\left(p_{k-2}, q_{k-2}\right)
\end{array}
$$

We now focus on computing vector relations between leaning points (upper and lower) inside a pattern. In the following we consider a DSS $(a, b, 0)$ in the first octant starting at the origin and ending at its second lower leaning point (whose coordinate along the $x$-axis is positive). We define $a / b=z_{n}=\left[0, u_{1}, \ldots, u_{n}\right]$ for some $n$. Points will be called $U_{1}, L_{1}, U_{2}$ and $L_{2}$ as shown in Fig. 11. We can state $\mathbf{U}_{1} \mathbf{L}_{1}=\mathbf{U}_{2} \mathbf{L}_{2}$ and $\mathbf{U}_{1} \mathbf{U}_{2}=\mathbf{L}_{1} \mathbf{L}_{2}=(b, a)$. We recall that the Freeman moves of $\left[U_{1} L_{1}\right]$ are the same as those of $\left[U_{2} L_{2}\right]$. Furthermore $\left[C_{U_{1}} C_{U_{2}}\right]$ form the pattern $(a, b)$ and $\left[C_{L_{1}} C_{L_{2}}\right]$ form the reversed pattern $(a, b)$. 


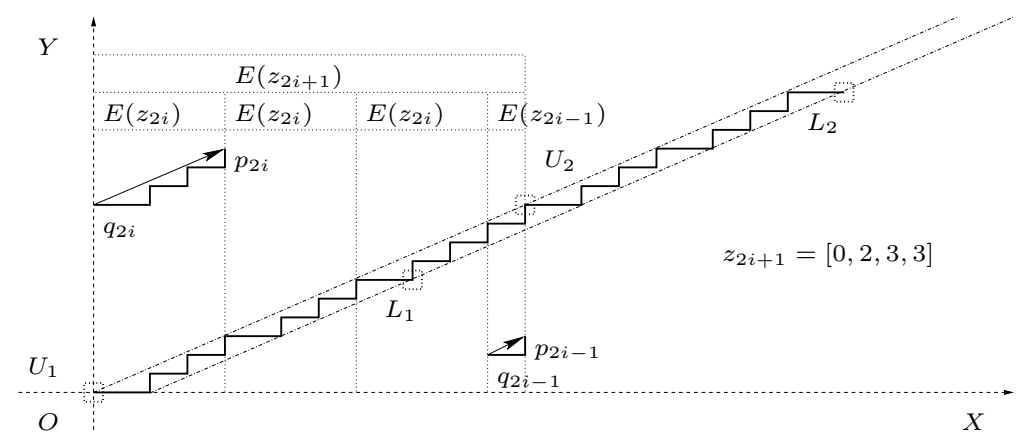

Fig. 1. A $\operatorname{DSS}(a, b, 0)$ with an odd complexity slope, taken between origin and its second lower leaning point

Proposition 1. A pattern with an odd complexity $($ say $n=2 i+1)$ is such that $\mathbf{U}_{\mathbf{1}} \mathbf{L}_{\mathbf{1}}=\left(u_{2 i+1}-1\right)\left(q_{2 i}, p_{2 i}\right)+\left(q_{2 i-1}, p_{2 i-1}\right)+(1,-1)$ and $\mathbf{L}_{\mathbf{1}} \mathbf{U}_{\mathbf{2}}=\left(q_{2 i}-1, p_{2 i}+1\right)$. Moreover the DSS $\left[U_{1} L_{1}\right]$ has $E\left(z_{2 i}\right)^{u_{2 i+1}-1}$ as a left factor, and the DSS $\left[L_{1} U_{2}\right]$ has $E\left(z_{2 i-1}\right)^{u_{2 i}}$ as a right factor.

Proposition 2. A pattern with an even complexity (say $n=2 i$ ) is such that $\mathbf{U}_{\mathbf{1}} \mathbf{L}_{\mathbf{1}}=\left(q_{2 i-1}+1, p_{2 i-1}-1\right)$ and $\mathbf{L}_{\mathbf{1}} \mathbf{U}_{\mathbf{2}}=\left(u_{2 i}-1\right)\left(q_{2 i-1}, p_{2 i-1}\right)+\left(q_{2 i-2}, p_{2 i-2}\right)+$ $(-1,1)$. Moreover the DSS $\left[U_{1} L_{1}\right]$ has $E\left(z_{2 i-2}\right)^{u_{2 i-1}}$ as a left factor, and the DSS $\left[L_{1} U_{2}\right]$ has $E\left(z_{2 i-1}\right)^{u_{2 i}-1}$ as a right factor.

\section{Properties of Maximal Segments for Convex Curves}

segments and digital edges of convex shape digitization. If $S$ is a subset of $\mathbb{R}^{2}$ its dilation by a real factor $r$ is denoted by $r \cdot S$. Let $\mathcal{D}_{m}$ be the digitization of step $1 / m: \mathcal{D}_{m}(S)=(m \cdot S) \cap \mathbb{Z}^{2}$. We call $\mathcal{L}^{1}$ the length estimator based on the city-block distance.

\subsection{Convex Digital Polygon (CDP)}

Definition 4. A convex digital polygon (CDP) $\Gamma$ is a subset of the digital plane equal to the digitization of its convex hull, i.e. $\Gamma=\mathcal{D}_{1}(\operatorname{conv}(\Gamma))$. Its vertices $\left(V_{i}\right)_{i=1 . . e}$ form the minimal subset for which $\Gamma=\mathcal{D}_{1}\left(\operatorname{conv}\left(V_{1}, \ldots, V_{e}\right)\right)$. The points on the boundary of $\Gamma$ form a 4-connected contour. The number of vertices (or edges) of $\Gamma$ is denoted by $n_{e}(\Gamma)$ and its perimeter by $\operatorname{Per}(\Gamma)$.

A $C D P$ is also called a lattice convex polygon [17. An edge is the Euclidean segment joining two consecutive vertices, and a digital edge is the discrete segment joining two consecutive vertices. It is clear that we have as many edges as digital edges and as vertices. From characterizations of discrete convexity [5], we clearly see that: 
Proposition 3. Each digital edge of a CDP is either a pattern or a succession of the same pattern whose slope is the one of the edge. In other words, both vertices are upper leaning points of the digital edge.

We now recall one theorem concerning the asymptotic number of vertices of CDP that are digitization of continuous shapes. It comes from asymptotic properties of random polytopes.

Theorem 1. (Adapted from Balog, Bárány [1]) If $S$ is a plane convex body with $\mathcal{C}^{3}$ boundary and positive curvature then $\mathcal{D}_{m}(S)$ is a CDP and

$$
c_{1}(S) m^{\frac{2}{3}} \leq n_{e}\left(\mathcal{D}_{m}(S)\right) \leq c_{2}(S) m^{\frac{2}{3}}
$$

where the constants $c_{1}(S)$ and $c_{2}(S)$ depend on extremal bounds of the curvatures along $S$. Hence for a disc $c_{1}$ and $c_{2}$ are absolute constants.

\subsection{Links Between Maximal Segments and Edges of CDP}

Maximal segments are DSS: between any two upper (resp. lower) leaning points lays at least a lower (resp. upper) leaning point. The slope of a maximal segment is then defined by two consecutive upper and/or lower leaning points. Digital edges are patterns and their vertices are upper leaning points (from Proposition 3). Thus, vertices may be upper leaning points but never lower leaning points of maximal segments. Moreover a maximal segment cannot be strictly contained into a digital edge.

We call supporting edge, a digital edge whose two vertices define leftmost and rightmost upper leaning points of a maximal segment.

Following lemma gives relations between maximal DSS and digital edges:

Lemma 1. (i) A supporting edge defines only one maximal segment: it is the only one containing the edge and it has the same slope. If a maximal segment contains two or more upper leaning points then there is a supporting edge linking its leftmost and rightmost upper leaning points with the same slope. If a maximal segment contains three or more lower leaning points then it contains a supporting edge.

(ii) If a maximal segment is defined by only two consecutive lower leaning points then its only upper leaning point is some vertex of the CDP by convexity.

Lengths of maximal segments and digital edges are tightly intertwined, as shown by the two next propositions (Proposition [5 follows from Proposition [1] and 2).

Proposition 4. Let $\left[V_{k} V_{k+1}\right]$ be a supporting edge of slope $\frac{a}{b}$ made of $f$ patterns $(a, b)$ and let $M S$ be the maximal segment associated with it (Lemma 1). Their lengths are linked by the inequality:

$$
\frac{1}{3} \mathcal{L}^{1}(M S) \leq \mathcal{L}^{1}\left(V_{k} V_{k+1}\right) \leq \mathcal{L}^{1}(M S) \leq \frac{f+2}{f} \mathcal{L}^{1}\left(V_{k} V_{k+1}\right)-2 \leq 3 \mathcal{L}^{1}\left(V_{k} V_{k+1}\right)
$$


Proposition 5. Let $M S_{k^{\prime}}$ be a maximal segment in the configuration of Lemma 1.ii, and so let $V_{k}$ be the vertex that is its upper leaning point. The length of the maximal segment is upper bounded by:

$$
\mathcal{L}^{1}\left(M S_{k^{\prime}}\right) \leq 4\left(\mathcal{L}^{1}\left(V_{k-1} V_{k}\right)+\mathcal{L}^{1}\left(V_{k} V_{k+1}\right)\right)
$$

A similar result related to linear integer programming is in [16]. It may also be obtained by viewing standard lines as intersection of two knapsack polytopes [10]. An elementary proof using pattern patterns is found in [6].

Theorem 2. Let $E$ be a supporting edge whose slope has a complexity $n, n \geq 2$, then the maximal segment containing $E$ includes at most $n$ other edges on each side of $E$.

Corollary 1. The shortest pattern of a supporting edge for which its maximal segment may contain $2 n+1$ digital edge is $z_{n}=[0,2, \ldots, 2]$. If the DCP is enclosed in a $m \times m$ grid, then the maximal number $n$ of digital edges included in one maximal segment is upper bounded as: $n \leq \log \frac{4 m}{\sqrt{2}} / \log (1+\sqrt{2})-1$.

Proof. The number $L=[0,2, \ldots, 2, \ldots]$ is a quadratic number equal to $-1+\sqrt{2}$. Its recursive characterization is $U_{n}=2 U_{n-1}+U_{n-2}$ with $U_{0}=0$ and $U_{1}=1$. Solving it leads to $U_{n}=\frac{\sqrt{2}}{4}\left((1+\sqrt{2})^{n}-(1-\sqrt{2})^{n}\right)$. Hence asymptotically, $U_{n} \approx \frac{\sqrt{2}}{4}(1+\sqrt{2})^{n}$ and $\lim _{n \rightarrow \infty} \frac{U_{n}}{U_{n+1}}=L$.

The shortest edge of slope complexity $n$ is clearly an $n$-th convergent of $L$. To fit into an $m \times m$ grid, the complexity $n$ is such that $U_{n+1} \leq m$. We thus obtain that $n \leq \log \frac{4 m}{\sqrt{2}} / \log (1+\sqrt{2})-1$.

\subsection{Asymptotic Number and Size of Maximal Segments}

We assume in this section that the digital convex polygon $\Gamma$ is enclosed in a $m \times m$ grid. We wish to compute a lower bound for the number of edges related to at least one maximal segment. We show in Theorem 3 that this number is significant and increases at least as fast as the number of edges of the DCP divided by $\log m$. From this lower bound, we are able to find an upper bound for the length of the smallest maximal segment of a DCP (Theorem 4). We first label each vertex of the DCP as follows: (i) a 2-vertex is an upper leaning point of a supporting edge, (ii) a 1-vertex is an upper leaning point of some maximal segment but is not a 2 -vertex, (iii) 0 -vertices are all the remaining vertices. The number of $i$-vertices is denoted by $n_{i}$. Given an orientation on the digital contour, the number of edges going from an $i$-vertex to a $j$-vertex is denoted by $n_{i j}$.

Theorem 3. The numbers of supporting edges and 1-vertices of $\Gamma$ are related to number of edges with

$$
\frac{n_{e}(\Gamma)}{\Omega(\log m)} \leq n_{1}+2 n_{22} .
$$

There are thus at least $n_{e}(\Gamma) / \Omega(\log m)$ maximal segments. 
Proof. From Theorem 2 and its Corollary 1, we know that a DSS hence a maximal segment cannot include more than $\Omega(\log m)$ edges. Hence there cannot be more than $\Omega(\log m)$ 0-vertices for one 1-vertex or for one 2 -vertex. We get $n_{00} \leq\left(n_{1}+n_{2}\right) \Omega(\log m)$. We develop the number of edges with each possible label: $n_{e}(\Gamma)=n_{22}+n_{02}+n_{12}+n_{20}+n_{21}+n_{00}+n_{01}+n_{10}+n_{11}$. Since, $n_{02}+n_{12} \leq n_{22}, n_{20}+n_{21} \leq n_{22}$ and $n_{01}+n_{10}+n_{11} \leq 3 n_{1}$, we get $n_{e}(\Gamma) \leq 3 n_{22}+n_{00}+3 n_{1}$. Noting that a 2 -vertex cannot be isolated by definition of supporting edges gives $n_{2} \leq 2 n_{22}$. Once inserted in $n_{00} \leq\left(n_{1}+n_{2}\right) \Omega(\log m)$ and compared with $n_{e}(\Gamma)$, we get the expected result.

We now relate the DCP perimeter to the length of maximal segments.

Theorem 4. The length of the smallest maximal segment of the DCP $\Gamma$ is upper bounded:

$$
\min _{l} \mathcal{L}^{1}\left(M S_{l}\right) \leq \Omega(\log m) \frac{\operatorname{Per}(\Gamma)}{n_{e}(\Gamma)} .
$$

Proof. We have $\operatorname{Per}(\Gamma)=\sum_{n_{e}} \mathcal{L}^{1}\left(E_{i}\right)$. We now may expand the sum on supporting edges (22-edges), on edges touching a 1-vertex, and on others. Edges touching 1-vertices may be counted twice, therefore we divide by 2 their contribution to the total length.

$$
\sum_{n_{e}} \mathcal{L}^{1}\left(E_{i}\right) \geq \sum_{n_{22}} \mathcal{L}^{1}\left(E_{j}^{22}\right)+\frac{1}{2} \sum_{n_{1}} \mathcal{L}^{1}\left(E_{k-1}^{? 1}\right)+\mathcal{L}^{1}\left(E_{k}^{1 ?}\right)
$$

For the first term, each supporting edge indexed by $j$ (a 22-edge) has an associated maximal segment, say indexed by $j^{\prime}$. From Proposition 4 we know that $\mathcal{L}^{1}\left(E_{j}^{22}\right) \geq \frac{1}{3} \mathcal{L}^{1}\left(M S_{j^{\prime}}\right)$. For the second term, each 1 -vertex indexed by $k$ is an upper leaning point of some maximal segment indexed by $k^{\prime}$. Proposition 5 holds and $\mathcal{L}^{1}\left(E_{k-1}^{? 1}\right)+\mathcal{L}^{1}\left(E_{k}^{1 ?}\right) \geq \frac{1}{4} \mathcal{L}^{1}\left(M S_{k^{\prime}}\right)$. Substitutions in Eq. (17) bring:

$$
\sum_{n_{e}} \mathcal{L}^{1}\left(E_{i}\right) \geq \frac{1}{3} \sum_{n_{22}} \mathcal{L}^{1}\left(M S_{j^{\prime}}\right)+\frac{1}{8} \sum_{n_{1}} \mathcal{L}^{1}\left(M S_{k^{\prime}}\right) \geq \frac{1}{8}\left(n_{1}+2 n_{22}\right) \min _{l} \mathcal{L}^{1}\left(M S_{l}\right)
$$

Inserting the lower bound of Theorem 3 concludes.

\section{Asymptotic Properties of Shapes Digitized at Increasing Resolutions}

We may now turn to the main interest of the paper: studying the asymptotic properties of discrete geometric estimators on digitized shapes. We therefore consider a plane convex body $S$ which is contained the square $[0,1] \times[0,1]$ (w.l.o.g.). Furthermore, we assume that its boundary $\gamma=\partial S$ is $\mathcal{C}^{3}$ with everywhere strictly positive curvature. This assumption is not very restrictive since people are mostly interested in regular shapes. Furthermore, the results of this section remains valid if the shape can be divided into a finite number of convex 
and concave parts; each one is then treated separately. The digitization of $S$ with step $1 / m$ defines a digital convex polygon $\Gamma(m)$ inscribed in a $m \times m$ grid. We first examine the asymptotic behavior of the maximal segments of $\Gamma(m)$, both theoretically and experimentally. We then study the asymptotic convergence of a discrete curvature estimator.

\subsection{Asymptotic Behavior of Maximal Segments}

The next theorem summarizes the asymptotic size of the smallest maximal segment wrt the grid size $m$.

Theorem 5. The length of the smallest maximal segment of $\Gamma(m)$ has the following asymptotic upper bound:

$$
\min _{i} \mathcal{L}^{1}\left(M S_{i}(\Gamma(m))\right) \leq \Omega\left(m^{1 / 3} \log m\right)
$$

Proof. Theorem 4 gives for the DCP $\Gamma(m)$ the inequality $\min _{i} \mathcal{L}^{1}\left(M S_{i}(\Gamma(m))\right) \leq$ $\Omega(\log m) \frac{\operatorname{Per}(\Gamma(m))}{n_{e}(\Gamma(m))}$. Since $\Gamma(m)$ is convex included in the subset $m \times m$ of the digital plane, its perimeter $\operatorname{Per}(\Gamma(m))$ is upper bounded by $4 m$. On the other hand, Theorem 1 indicates that its number of edges $n_{e}(\Gamma(m))$ is lower bounded by $c_{1}(S) m^{2 / 3}$. Putting everything together gives $\min _{i} \mathcal{L}^{1}\left(M S_{i}(\Gamma(m))\right) \leq$ $\Omega(\log m) \frac{4 m}{c_{1}(S) m^{2 / 3}}$ which is once reduced what we wanted to show.

Although there are points on a shape boundary around which maximal segments grow as fast as $O\left(\mathrm{~m}^{1 / 2}\right)$ (the critical points in 13 ), some of them do not grow as fast. A closer look at the proofs of Theorem 4 shows that a significant part of the maximal segments (at least $\Omega(1 /(\log m))$ ) has an average length that grows no faster than $\Omega\left(m^{1 / 3} \log m\right)$. This fact is confirmed with experiments. Fig. 2] left, plots the size of maximal segments for a disk digitized with increasing resolution. The average size is closer to $m^{1 / 3}$ than to $m^{1 / 2}$.

\subsection{Asymptotic Convergence of Discrete Geometric Estimators}

A useful property that a discrete geometric estimator may have is to converge toward the geometric quantity of the continuous shape boundary when the digitization grid gets finer [3, 4, 11].

We now recall the definition of a discrete curvature estimator based on DSS recognition [3].

Definition 5. Let $P$ be any point on a discrete contour, $Q$ and $R$ are the extremities of the longest DSS starting from $P$ (called half-tangents). Then the curvature estimator by circumcircle $\hat{\kappa}(P)$ is the inverse of the radius of the circle circumscribed to $P, Q$ and $R$, rescaled by the resolution $m$.

Experiments show that this estimator rather correctly estimates the curvature of discrete circles on average $(\approx 10 \%$ error $)$. It is indeed better than any other curvature estimators proposed in the litterature. Theorem B.4 of [3] demonstrates the asymptotic convergence of this curvature estimator, subject to the conjecture: 

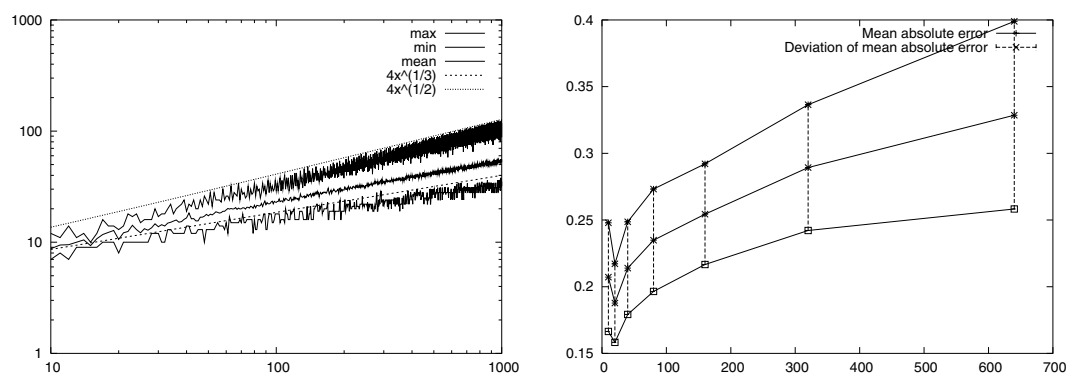

Fig. 2. For both curves, the digitized shape is a disk of radius 1 and the abscissa is the digitization resolution. Left: plot in log-space of the $\mathcal{L}^{1}$-size of maximal segments. Right: plot of the mean and standard deviation of the absolute error of curvature estimation, $|\hat{\kappa}-1|$ (expected curvature is 1$)$

Conjecture 1. 3] Half-tangents on digitized boundaries grow at a rate of $\Omega\left(m^{1 / 2}\right)$ with the resolution $m$.

However, with our study of maximal segments, we can state that

Claim. Conjecture 1 is not verified for digitizations of $\mathcal{C}^{3}$-curves with strictly positive curvature. We cannot conclude on the asymptotic convergence of the curvature estimator by circumcircle.

Proof. It is enough to note that half-tangents, being DSS, are included in maximal segments and may not be longer. Furthermore, since maximal segments cover the whole digital contour, some half-tangents will be included in the smallest maximal segments. Since the smallest maximal segments are no longer than $\Omega\left(m^{1 / 3} \log m\right)$ (Theorem 5 ), the length of some half-tangents has the same upper bound, which is smaller than $\Omega\left(m^{1 / 2}\right)$.

The asymptotic convergence of a curvature estimator is thus still an open problem. Furthermore, precise experimental evaluation of this estimator indicates that it is most certainly not asymptotically convergent, although it is actually on average one of the most stable discrete curvature estimator (see Fig. 2, right). Former experimental evaluations of this estimator were averaging the curvature estimates on all contour points. The convergence of the average of all curvatures does not induce the convergence of the curvature at one point.

\section{Conclusion}

We show in this paper the relations between edges of convex hulls and maximal segments in terms of number and sizes. We provide an asymptotical analysis of the worst cases of both measures. A consequence of the study is the refutation of an conjecture related to the asymptotic growth of maximal segments and which was essential in proving the convergence of a curvature estimator based 
on DSS and circumcircles [3. Our work also applied to digital tangents since their convergence relies on the same conjecture. The existence of a convergent discrete estimator of curvature based on DSS is thus still a challenging problem and we are currently investigating it.

\section{References}

1. Antal Balog and Imre Bárány. On the convex hull of the integer points in a disc. In SCG '91: Proceedings of the seventh annual symposium on Computational geometry, pages 162-165. ACM Press, 1991.

2. J. Berstel and A. De Luca. Sturmian words, lyndon words and trees. Theoret. Comput. Sci., 178(1-2):171-203, 1997.

3. D. Coeurjolly. Algorithmique et géométrie pour la caractérisation des courbes et des surfaces. PhD thesis, Université Lyon 2, Décembre 2002.

4. D. Coeurjolly and R. Klette. A comparative evaluation of length estimators of digital curves. IEEE Trans. on Pattern Anal. and Machine Intell., 26(2):252-257, 2004.

5. Chul E.Kim. Digital convexity, straightness, and convex polygons. IEEE Trans. on Pattern Anal. and Machine Intell., 6(6):618-626, 1982.

6. J.-O. Lachaud F. de Vieilleville and F. Feschet. Maximal digital straight segments and convergence of discrete geometric estimators. Research Report 1350-05, LaBRI, University Bordeaux 1, Talence, France, 2005.

7. F. Feschet and L. Tougne. Optimal time computation of the tangent of a discrete curve: application to the curvature. In Discrete Geometry and Computer Imagery (DGCI), volume 1568 of $L N C S$, pages 31-40. Springer Verlag, 1999.

8. F. Feschet. and L. Tougne. On the Min DSS Problem of Closed Discrete Curves. In A. Del Lungo, V. Di Gesù, and A. Kuba, editors, IWCIA, volume 12 of Electonic Notes in Discrete Math. Elsevier, 2003.

9. G.H. Hardy and E.M. Wright. An introduction to the theory of numbers. Oxford University Press, fourth edition, 1960.

10. A. S. Hayes and D. C. Larman. The vertices of the knapsack polytope. Discrete Applied Mathematics, 6:135-138, 1983.

11. R. Klette and J. Žunić. Multigrid convergence of calculated features in image analysis. Journal of Mathematical Imaging and Vision, 13:173-191, 2000.

12. V. Kovalevsky and S. Fuchs. Theoretical and experimental analysis of the accuracy of perimeter estimates. In Förster and Ruwiedel, editors, Proc. Robust Computer Vision, pages 218-242, 1992.

13. J.-O. Lachaud. On the convergence of some local geometric estimators on digitized curves. Research Report 1347-05, LaBRI, University Bordeaux 1, Talence, France, 2005.

14. J.-O. Lachaud, A. Vialard, and F. de Vieilleville. Analysis and comparative evaluation of discrete tangent estimators. In E. Andrès, G. Damiand, and P. Lienhardt, editors, Proc. Int. Conf. Discrete Geometry for Computer Imagery (DGCI'2005), Poitiers, France, LNCS. Springer, 2005. To appear.

15. J.-P. Réveillès. Géométrie discrète, calcul en nombres entiers et algorithmique. Thèse d'etat, Université Louis Pasteur, Strasbourg, 1991.

16. V. N. Shevchenko. On the number of extreme points in linear programming. Kibernetika, 2:133-134, 1981. In russian.

17. K. Voss. Discrete Images, Objects, and Functions in $\mathbb{Z}^{n}$. Springer-Verlag, 1993. 